\title{
Comparing league formats with respect to match importance in Belgian football
}

\author{
Dries R. Goossens · Jeroen Beliën · Frits \\ C.R. Spieksma
}

Received: date / Accepted: date

\begin{abstract}
Recently, most clubs in the highest Belgian football division have become convinced that the format of their league should be changed. Moreover, the TV station that broadcasts the league is pleading for a more attractive competition. However, the clubs have not been able to agree on a new league format, mainly because they have conflicting interests. In this paper, we compare the current league format, and three other formats that have been considered by the Royal Belgian Football Association. We simulate the course of each of these league formats, based on historical match results. We assume that the attractiveness of a format is determined by the importance of its games; our importance measure for a game is based on the number of teams for which this game can be decisive to reach a given goal. Furthermore, we provide an overview of how each league format aligns with the expectations and interests of each type of club.
\end{abstract}

Keywords tournament design $\cdot$ match importance $\cdot$ football $\cdot$ simulation $\cdot$ optimization

Dries R. Goossens

PostDoc researcher for Research Foundation - Flanders,

Center for Operations Research and Business Statistics (ORSTAT),

Faculty of Business and Economics - K.U.Leuven,

Naamsestraat 69, 3000 Leuven, Belgium

E-mail: dries.goossens@econ.kuleuven.be

Jeroen Beliën

Research Center for Modelling and Simulation - H.U.Brussel

Stormstraat 2, 1000 Brussels, Belgium

Affiliated researcher Operations Management Group

Department of Decision Sciences and Information Management

Faculty of Business and Economics - K.U.Leuven

Naamsestraat 69, 3000 Leuven, Belgium

E-mail: jeroen.belien@hubrussel.be

Frits C.R. Spieksma

Center for Operations Research and Business Statistics (ORSTAT),

Faculty of Business and Economics - K.U.Leuven,

Naamsestraat 69, 3000 Leuven, Belgium

E-mail: frits.spieksma@econ.kuleuven.be 


\section{Introduction}

For decades, the first (and highest) division in Belgian football (also known as the "Jupiler League"), has been organized as a double round robin tournament, i.e., a tournament in which each team plays each other team twice, once at home and once away. The games of the tournament are grouped in rounds, such that each team plays exactly once per round. Since the season 1977-1978, 18 teams take part in this competition, and only two changes have been implemented since. From the season 1995-1996 onwards, three points are awarded for a win instead of two. Second, since the season 2005-2006, the team that ends second from bottom in the competition no longer relegates directly, but gets a chance to maintain its place in the first division in a double round robin tournament with three teams from second division. However, during the last years, most clubs in the Jupiler League have become convinced that changes in the way the competition is played are needed.

Despite the fact that most teams are convinced of the necessity of change, there is little agreement on what the changes should be, since arguments and preferences of the teams depend on their (aspired) role in the competition. We can classify the teams involved into four categories. The first category consists of the traditional top teams. These teams (RSC Anderlecht, Club Brugge, Racing Genk, and Standard Liège) are the main contenders for the title of league champion nearly every season, and only top teams managed to become the league champion over the last 10 years. The traditional top teams are especially looking for a stronger competition (Hauspie, 2007). They reason that by playing more top level games, they will be able to increase their budget and attract and train better players. On the other hand, they would like to play less games overall, in order to avoid overtired players, when the regular season is to be combined with a European campaign. This should increase their chances in Europa League or Champions League football, where Belgian teams have not been excelling recently. A second category is what we call the ambitious middle group. These six teams (Gent, Charleroi, Germinal Beerschot, Lokeren, Westerlo, and Mouscron) have been continuously present in the first division during the last 10 years. These teams are looking for a competition format that preserves or improves their chances for a European ticket, or even a league title (Colin, 2007). The third group is what we call the small six. These teams are mainly concerned with holding on to their place in the league, and thus are not at all fond of reducing the number of competitors, or increasing the number of teams that relegate to the second division. Finally, a fourth group consists of 10 teams that we call the second division teams. These teams are those teams that were playing in or relegated to the second division in the season 2007-2008, but are anxious to promote to the first division. Some of these teams have quite a history in the first division, while others come from lower divisions, and are very ambitious to continue their rise (De Vos, 2008b). Apart from the clubs, the TV station that broadcasts the competition is looking for a league format that is as attractive as possible. In other words, the TV station wants to avoid matches that have no importance at all, since these are the games that do not attract viewers. Given the fact that the money from the broadcasting contract is the main source of income for many clubs, the wishes of the TV station carry a considerable weight (De Vos, 2008a). 


\section{Overview of the approach}

In order to quantitatively and objectively analyze the different league formats, we developed a unique, integrated approach that combines both simulation and optimization. Simulation is needed to generate the outcomes of the matches played in a particular league format. Optimization is required both to develop an acceptable match schedule for each league format and to determine the importance of a match. Analyzing a particular league format involves repeating the following steps for a large number of seasons.

1. Use optimization to develop a suitable match schedule given the teams in the league.

2. For all rounds in the regular competition:

(a) Use optimization to determine the importance of each match.

(b) Simulate all match outcomes.

3. Simulate the play-offs (if present in the league format).

4. Replace the relegated clubs by the climbers from the second division.

In section 3, we discuss a number of league formats that have been considered to be adopted for the Belgian Jupiler League. Specifics on the way the season schedule is created for each of the formats is provided in section 4 . In section 5 , we explain how we simulated match results. Our measure of match importance is detailed in section 6 ; in section 7 , we compare the various league formats using this measure. Moreover, we simulate what teams from each category may expect from the various league formats with respect to their chances for the league title, European football, or relegation.

\section{Reforming the Belgian football league}

There are numerous ways to organize a competition, even if we limit ourselves to those formats that are common in football. The World Cup, the Champions League, and the Europa League are currently organized as a two stage event, where the first stage consists of a round robin tournament (single for the World Cup, double for the Europa League and the Champions League), and the second stage is an instant knock-out tournament, with two games in any round but the final for the Champions League and the Europa League, and just one for the World Cup. More than a decade ago, Griggs and Rosa (1996) published an overview of competition formats that were used then in European national leagues. At that time, the large majority of the football competitions were organized as a double round robin tournament, using so-called canonical schedules (see e.g., de Werra (1980)). Nowadays, most European countries still use a double round robin tournament (e.g., Germany (Bartsch et al., 2006), Italy (Della Croce and Oliveri, 2006), and England (Kendall, 2008)) although some countries (e.g., The Netherlands) have introduced a play-off stage after the regular competition, in order to decide on relegation or qualification for the European football tournaments.

In some countries, the competition consists of a single round robin tournament. Since in this tournament, each team meets each other team only once, the number of home games and the opponents in these games strongly determines the fairness of the schedule (in a double round robin tournament, this is obviously not an issue). Denmark is one of the few countries that have a triple round robin tournament (i.e., teams meet 
three times). This format also leads to an uneven distribution of home and away games (Rasmussen, 2008). In Austria, a quadruple round robin tournament is played with 10 teams, resulting in a schedule with 36 rounds (Bartsch et al., 2006). One of the more peculiar competition formats can be found in Chile, where 20 teams are organized into 4 groups of 5, although they all play once against each of the other 19 teams. After this single round robin tournament, the best two teams of each group advance to a play-off stage, where the actual league champion is decided (see Durán et al. (2007)). For more information on scheduling sports competitions, we refer the reader to an annotated bibliography by Kendall et al. (2010). In the remainder of this section, we discuss the way in which Belgium's Jupiler League is organized currently, followed by three formats that were under consideration by the Royal Belgian Football Association to be used for the season 2009-2010.

Currently, the Jupiler League is played as a straightforward double round robin tournament with 18 teams, spread over 34 rounds. The winner of this league is the champion, and qualifies for the (qualification stage of the) Champions League. The second in the league can also try to qualify for the Champions League, whereas the third and the fourth in the league go to the Europa League. The team that ends up last relegates to the second division. The team that ends up on the second to last place can however remain in the highest division if it wins a double round robin tournament with three second division clubs. This means that 12 additional games are played after the regular competition, which consists of 306 matches (Goossens and Spieksma, 2009).

The first alternative that we consider is loosely based on the competition in The Netherlands, where since the 2005-2006 season, a post-season play-off has been introduced to determine the league champion, relegation, and qualification for the European tournaments (see e.g., Koning (2007)). The format is a double round robin tournament with 16 teams, where the first in the league is the champion, and the last relegates to the second division. Two play-off tournaments decide which teams qualify for European football, and which teams are relegated. The European play-offs are played with the teams ranked 2 to 5 in the league: in a first stage with a home and away game, the second ranked team plays the fifth ranked team, while the third ranked team meets the fourth ranked team. The second stage consists of two games between the winners, and two games between the losers of these games. The winners play against each other in order to decide which team can qualify for the Champions League; the losing team goes to the Europa League. The losers play for the last Europa League ticket. The relegation play-offs are played with the teams ranked 14th and 15th, and 6 teams from the second division. These teams play a direct knock-out tournament, with a home and an away game in each stage, until two teams remain. The regular competition, together with the European play-off results in 248 games per season; the promotion/relegation tournament consists of another 14 matches. Since almost all outcomes in this competition are decided by a play-off stage, we will refer to it as the play-off league. Notice that many variants of this format exist, for instance with a play-off deciding on the league title, or a setting in which teams that ended up in the middle of the ranking still have a chance to qualify for European football (see e.g., Geril (2008b)).

The second league format splits the competition into two parts: an autumn competition and a spring competition. Each of these competitions consists of two series, $\mathrm{A}$ and $\mathrm{B}$, of 10 teams each, that play a double round robin tournament. The com- 
petition starts with the autumn competition, consisting of 18 rounds. The winner of the A series of this competition qualifies for European football (Champions League qualification). The best five teams of the B series replace the worst five teams in the A series of the subsequently played spring competition, which is again a double round robin tournament for both the $\mathrm{A}$ and the $\mathrm{B}$ series. The winner of the $\mathrm{A}$ series in the spring competition is the league champion, and as such, qualifies for European football (Champions League qualification). The two worst teams in the B series relegate directly to the second division. The final tickets for European football (Europa League) are awarded to the winners of a play-off tournament, played among the 4 teams that were second in the A series or first in the B series in the autumn or the spring competition. If any of these 4 teams would already have qualified for European football in some other way, it is replaced by the next in line from the subcompetition it qualified from. First, the two B series winners play against each other in a single game with home advantage for the spring winner. Next, the winner meets the team that ended second in the spring A series, in a single game at the ground of the second from the A series. The winner of this game qualifies for the Europa League. The loser then challenges the second in the autumn A series for the final ticket for European football, again in a single game with home advantage for the second in the autumn series. For the next season, the worst five teams of the A series are again replaced by the best five teams of the B series, and two teams promote from the second division, replacing the two last of the spring B series. With this new composition, the autumn competition of the next season can start. This league format has 363 games in total, and is referred to as the Wijnants league, named after its inventor Herman Wijnants, chairman of the football club Westerlo VV. With his league format, Wijnants attempted to find a compromise, reconciling the various clubs with conflicting interests. We refer to Hauspie (2007) for more details on the Wijnants league. This format has a number of variants as well, involving e.g., series of 9 or 12 teams instead of 10 , or limiting the number of teams that promote from series B to A to three or four.

Finally, the third league format also consists of two stages, but involves only 16 teams. The season starts with a double round robin tournament, consisting of 30 rounds. The last team in this tournament directly relegates to the second division. The team that ends 15 th will play a double round robin tournament with 3 second division teams for a place in the first division. The first 6 teams of the first stage tournament advance to another double round robin tournament, for which they can keep half of the points they collected in the first stage. The winner of this tournament is the league champion, and qualifies for the Champions League. The second also qualifies for the (qualification stage of) the Champions League, and the third has earned a ticket for the Europa League. The teams ranked 7 to 12 also play a double round robin tournament for which they can keep half of the points they earned in the first stage of the competition. The winner of this tournament plays twice (home/away) against the fourth of the tournament with teams ranked 1 to 6 twice, to determine which team gets the final Europa League ticket. In this league format, most teams will play 40 rounds, and at the end of the season 342 games will have been played (apart from 12 matches in the promotion/relegation tournament). We refer to this league as the future league (see also (Geril, 2008a)). 
4 Scheduling the league formats

In this section we describe how the schedules for each of the competition formats were constructed. In Belgium, the wishes of the various stakeholders are collected through the calendar committee, which is responsible for creating an acceptable schedule. For reasons of fairness, the committee is convinced that teams should not play more than two consecutive home (away) matches. When a team has two consecutive home matches, or two consecutive away matches, we say it has a break. Although breaks are obviously unavoidable, the committee thinks it is imperative that no team starts or ends the competition with a break, and that the total number of breaks over all teams should be minimal. As in many countries, in Belgium it is considered fair to schedule the second half of the competition identically to the first half, but with the home advantage inverted (i.e., mirroring). Since most teams prefer not to play against all traditional top teams consecutively, the calendar committee asked that no team would have to play more than twice against a top team in four consecutive games. Furthermore, the calendar committee stated that every team should receive a top team at home at least once in each half of the season. The TV station that broadcasts the games also has a number of wishes regarding the schedule. First of all, they want for each round at least one (and preferably two) of the four top teams to play an away game. The underlying motivation is that a top team's home games are less interesting, since the top team tends to win these games without much effort. Further, they wish to have at most one game between top teams per round, and no top games in the first four rounds. Moreover, the top games should be spread over the season. Apart from these demands, there are still a number of wishes from the clubs or the police that we do not take into account, since they are valid for only one specific season (e.g., no home game for some team on a given round, because of some other event taking place in the city).

Satisfying all demands turned out to be impossible since they were conflicting. Therefore, we asked the calendar committee to attach one of five priority levels to each of the wishes. We linked a penalty with each priority level, such that this penalty is incurred if the corresponding constraint is violated. The goal is then to find a schedule with minimal incurred penalties. Since this scheduling problem is computationally too demanding, we decompose it into two subproblems. In the first phase, each team is assigned a home-away pattern, which indicates when a team plays a home game, and when it plays away. In the second phase, the actual opponents are decided, given the home-away pattern for each team. For more details on the scheduling of the Jupiler League in its current format, we refer to Goossens and Spieksma (2009).

Although of course, it is not yet clear what the wishes of the calendar committee would be when confronted with a different league format, we think we can safely assume that the demands mentioned above would still be valid. Therefore, we use the same criteria and method to develop schedules for the play-off league, the Wijnants league, and the future league. 


\section{Simulating match results}

In the literature, there are two main approaches to model the outcomes of matches in football. The first approach (see e.g., Dixon and Coles (1997) or Dixon and Pope (2004)) uses bivariate Poisson regression to estimate forecasting models for goals scored and conceded. Forecasts of the match outcome (win-draw-loss) can be derived indirectly, by aggregating the estimated probabilities assigned to appropriate permutations of goals scored and conceded by the two teams. In the second approach (see e.g., Koning (2000), Forrest and Simmons (2000), Audas et al. (2002) or Goddard and Asimakopoulos (2004)), the match outcomes are predicted directly by using discrete choice regression models like ordered probit regression. Goddard (2005) presents an extensive study in which the forecasting power of both approaches are compared using a 25-year data set on English league football match outcomes. Although the first approach is favored by most applied statisticians, according to Goddard's study, the differences between the forecasting performance of both approaches appear to be relatively small. Karlis and Ntzoufras (2003) present an alternative approach that combines issues of both the (indirect) goals based models and the (direct) outcomes based models as they try to predict the difference between the goals made by the two teams.

The objective of this study is to evaluate different competition formats rather than predicting match results. Nevertheless, in order to simulate, in a reliable way, all matches in a particular competition format, our analysis relies heavily on the estimated probabilities of match outcomes. Since the number of goals scored and conceded is of less importance, we adopted the second approach that directly models the three possible match results (win-draw-loss). These trinomial probability distributions have been estimated for each match starting from 10 years historical data, including all matches of season 1998-1999 till season 2007-2008. If the result of a particular match, say team A versus team B, was five times a win for team A, two times a draw, and three times a win for team B, the resulting probability distribution for that match would be: $50 \%$ chance team A wins, $20 \%$ chance on a draw, and $30 \%$ chance team B wins. Remark that we distinguish between the matches $\mathrm{A}$ versus $\mathrm{B}$ and $\mathrm{B}$ versus $\mathrm{A}$, and thus take into account the home advantage.

Additionally, we developed probability distributions for the matches of each team against the newly promoted teams from the second division. Note that several different teams contribute to the probability distributions of these matches, since the newly promoted teams tend to differ over the seasons. The advantage of this approach is twofold. First of all, it enables us to simulate the result of a future match between a team and a newly promoted team (which could be a match that has never been played before in the first division). Second, it allows us to construct more reliable probability distributions for those matches that were played less than 4 times over the past 10 years. Indeed, due to lack of data, the resulting probability distributions for these matches are very unreliable. For such matches, we replaced the team that played the least matches in the Jupiler League over the past 10 years with one of the two in that season just promoted teams (a random choice) and considered the probability distributions of the match results between this new team and the other team. Note that the replacing team (the first or the second climber) always played 10 seasons, since there were always two climbers. Hence, the resulting distributions are deduced from 10 matches. In case both teams played less than four seasons, both teams were replaced by the first and second 
climber (which team is replaced by which climber is a random choice). The assumption here is that all teams that played less than four seasons in the first division over the past 10 years are equal with respect to their chances to win, draw or loose a particular match and that these chances are equal to the chances of a newly promoted team. Our results on the predicting accuracy of match results (see further) seem to justify this assumption.

The approach described above leaves three important issues out of consideration. First, the current approach ("basic") considers all played matches over the past 10 years to be equally important. It seems to make sense, however, to give a higher weight to more recent matches when calculating the probability distributions. To take into account this issue, we constructed a second set of distributions ("recent") in which we assign a weight of 3 to the matches of the three most recent seasons, a weight of 2 to the matches of 4 to 6 seasons ago, and a weight of 1 to the matches of 7 to 10 seasons ago.

Second, by solely looking at match outcomes (win-draw-loss), the differences between goals scored and goals conceded is completely ignored. However, it can be argued that a significant win should get a higher weight in deriving the probability distributions than a close win. Similarly, a significant loss should have a larger weight than a small loss. Therefore, we constructed a third set of distributions ("dif3") in which a win (loss) with three or more goals difference was assigned a weight equal to 3 , while the other (small) wins and losses had a weight equal to 1 . We also combined this setting with the setting in which we gave a higher weight to recent matches, resulting in a fourth set of distributions ("recent_dif3").

Third, it is reasonable to assume that the results of matches are not independent, and that a team that has won a series of matches (a hot streak), is more likely to win the next match as well. Taking the form of the teams into account could well increase the forecasting accuracy. Nevertheless, we chose to ignore the form effect in the simulation experiments described hereafter. The reason is twofold. First, recall that the objective in this paper is not to forecast individual match outcomes, but to simulate, as reliably as possible, a whole championship. If we would take into account the form effect when simulating the match outcomes, the final ranking could be affected too heavily by the outcomes of the first matches. Indeed, a number of lucky wins in the beginning of the championship results in a positive form effect, increasing the chances to win the next matches, which, again reinforces the form effect. Second, preliminary results (see Goossens et al. (2008)) indicated that the form effect is very small in the Jupiler League. Therefore, we believe it is justified to exclude the form effect from our simulations.

It must be clear that the results of our simulation studies heavily depend on the quality of the match outcome distributions. In a first attempt to validate these probability distributions, we checked the accuracy in predicting the correct outcomes of individual matches. Therefore, we derived the distributions as described above, but this time based on the historical match outcomes from season 1997-1998 till season 2006-2007 and evaluated these distributions with respect to the forecasting accuracy on the results of all 306 matches played in season 2007-2008. For each match we predicted the result corresponding with the highest probability in the trinomial distribution. For 
instance, in the example above, we would predict team A to win. We applied the accuracy check for the four different scenarios of generating the distributions, described earlier. In addition, the accuracy check was performed for two simple heuristics: random match outcomes and always predict a home win. Table 1 contains the results.

Table 1 Accuracy of predicting the match outcomes of the 2007-2008 first division football league using match outcome distributions derived from seasons 1997-1998 till 2006-2007

\begin{tabular}{rr}
\hline & Accuracy \\
\hline basic & $46.4 \%$ \\
recent & $44.8 \%$ \\
dif3 & $47.1 \%$ \\
recent_dif3 & $46.4 \%$ \\
random & $29.7 \%$ \\
always home win & $46.4 \%$ \\
\hline
\end{tabular}

Compared with the more complex models presented in the literature where accuracies between $40 \%$ and $50 \%$ are reported (see, e.g., McHale and Scarf (2006)), our simple approach based solely on historical match results performs, at first sight, remarkably well. Based on the accuracies reported in Table 1, one might conclude that the "dif3"-distributions are most suited for the league simulations. However, observe that, since $46.4 \%$ of the matches of the $2007-2008$ season ended in a home win, the extremely simple "always predict a home win"-rule results in an accuracy of $46.4 \%$ ! However, if these distributions would be used as input for our simulations, each team would always have the same number of points in the final ranking, which is obviously not realistic.

This last observation indicates that the forecasting accuracy of individual match outcomes, as reported in Table 1, is not the most appropriate criterion to select the distributions. Indeed, in order to evaluate the 4 types of distributions, we need to measure their ability of simulating reliable championships, which is slightly different from predicting individual match outcomes. The literature on forecasting championship results is much sparser than the literature on predicting individual match outcomes. Silva et al. (2002) present a Monte Carlo simulation model to analyze the Brazilian National Soccer Championship in which they determine the necessary points to achieve classification for the play-offs and to avoid relegation, as a function of the number of teams in the championship. Unfortunately, they assume that each participant is equally strong, i.e., each team has the same chances of winning a match. Their model is validated through comparison of the simulated number of draws with the real number of draws. Obviously, since our model works with different win-draw-loss probabilities for each match, this type of validation would not suffice. Koning et al. (2003) developed a simulation model to identify the team that is most likely to win a football tournament. The model is tested by comparing the predictions with the actual results of the World Cups 1994 and 1998 and the European Cups 1996 and 2000. Since our model not only focusses on the championship winner, but on the results of all participants, this type of validation would not suffice either. Smyth and Smyth (1994) predict the American Baseball division rankings using data on the players salaries. The forecasts are com- 
pared with the final rankings by calculating the correlation coefficients between the ranks. This type of validation would be appropriate for our setting and, therefore, we used a similar measure, but besides the team ranks, the points obtained per team in the final ranking were also validated. However, instead of the correlation coefficients, we calculated the average differences between both the ranks and the total points obtained by each team in the simulated championship and the real ranks and points of the final ranking of 2007-2008. The reason is that these numbers are much easier to interpret than the correlation coefficients. Table 2 presents the results for the four types of distributions.

Table 2 Accuracy of predicting the final ranking of the 2007-2008 first division football season using match outcome distributions derived from the seasons 1997-1998 till 2006-2007

\begin{tabular}{rrr}
\hline & Avg. difference in points per team & Avg. difference in rank per team \\
\hline basic & 9.80 & 3.67 \\
recent & 9.64 & 3.60 \\
dif3 & 10.37 & 3.69 \\
recent_dif3 & 9.87 & 3.59 \\
random & 12.98 & 5.95 \\
always home win & 12.78 & 4.56 \\
\hline
\end{tabular}

Table 2 indicates that the best results are obtained using the "recent"-distributions, since these distributions result in the best score with respect to difference in points and a second (but very close to) best score with respect to the difference in ranks. Therefore, the "recent"-distributions are used in the simulations. Note that these distributions clearly outperform the random and the "always predict a home win"-distributions.

\section{Measuring match importance}

The importance of a game is useful not just for comparing various tournament designs, but also for selecting games to broadcast, assigning referees, or explaining the attendance. Logically, the concept of match importance has been discussed before in a number of papers, and various rather simple measures have been developed. Jennett (1984) measures the importance of a game with respect to winning the league as the inverse of the number of remaining games that still need to be won. When a team can no longer win the league, the measure gets the value 0. Audas et al. (2002) call a game important if it is possible for either of the opponents to still win the league (or relegate), if all other teams draw in the rest of their games. The most commonly accepted measure for match importance however, is what Schilling (1994) calls the conditional importance $S_{i}(X)_{t, t+k}$ of match at time $t+k$ for a team $i$ at time $t$ with respect to outcome $X$, and is defined as follows.

$$
S_{i}(X)_{t, t+k}=p\left(X_{i} \mid W_{i, t+k}, H_{t}\right)-p\left(X_{i} \mid L_{i, t+k}, H_{t}\right)
$$

We use the notation $X_{i}$ for an outcome $X$ that is achieved by team $i$. This outcome may be the league championship, but just as well qualification for European football or relegation. The event where team $i$ wins its game scheduled at time $t+k$ 
is represented by $W_{i, t+k}$; the event of team $i$ losing this game by $L_{i, t+k}$. Finally, $H_{t}$ represents the history of games that have already been played at time $t$. Note that this measure does not take into account a draw; extending this definition to include draws is not straightforward. The probabilities in the Schilling measure are however usually determined using a Monte Carlo simulation (see Scarf and Shi (2008), who use the Schilling measure in this way and apply it to the games in the English Premier League).

We propose to measure the attractiveness of a competition format through match importance. We define the importance $I_{m}(X)$ of a match $m$ with respect to outcome $X$ as

$$
I_{m}(X)= \begin{cases}\frac{2 n_{m}(X)}{A_{m}(X)^{2}} & \text { if } A_{m}(X)>0 \\ 0 & \text { if } A_{m}(X)=0\end{cases}
$$

where $A_{m}(X)$ is the number of teams that are still competing for outcome $X$ at the time when match $m$ is played, and $n_{m}(X)$ is the number of teams in match $m$ that are still competing for outcome $X$. We scaled this measure with a factor 2 , to make sure that the importance of a match coincides with the interval $[0,1]$.

With our measure, the importance of a match with respect to some outcome increases when less teams are in the running for that outcome (provided of course that there are still teams competing for that outcome). Indeed, when only 2 teams are in the running for e.g., the league championship, their games will be highly important. If one team loses its game, it could be enough that its competitor wins to decide the championship. Contrarily, when a team is still in the race for the championship together with 5 other teams, the fact whether or not this team will be league champion will depend on the results of many other games, thus reducing the importance of this team's game. Furthermore, we consider a match to be unimportant with respect to some outcome, if it can have no influence on the outcome for any of the competing teams. The underlying idea is that a game between two teams that have nothing to gain or to lose is no longer interesting for a TV station to broadcast, and will attract less fans to the stadium. Bojke (2007) confirms that this is the case in the English Premier League. Of course, we do not claim that unimportant games cannot be a tremendously spectacular, but beforehand, they will typically not attract big crowds. In the first round of the season, no game will be unimportant, because at that time any team can become the league champion, even though the chances may be very slim. Nevertheless, according to our measure, the importance of these games will be limited. Finally, we point out that our approach is in line with the Schilling measure (1), since when we find through optimization that a match is unimportant, this means that $p\left(X_{i} \mid W_{i, t+k}, H_{t}\right)=0$ and thus $S_{i}(X)_{t, t+k}=0$. Notice however that matches for which $S_{i}(X)_{t, t+k}=0$ according to a Monte Carlo simulation need not be unimportant using our optimization approach.

In order to compute $A_{m}(X)$, the number of teams that are still competing for outcome $X$ at the time when match $m$ is played, we need to know for each team $t$ the highest and the lowest ranking that team can still reach at the end of the season. We use the following notation. We define $T$ as the set of teams in the competition, and $G(q)$ as the set of games that are yet to be played, given that there are $q$ rounds remaining. We define the variable $w_{i j}$ to be 1 if $i$ wins its home game against $j$, and 0 otherwise. Furthermore, we say that $l_{i j}$ is 1 if $i$ loses its home game against $j$, otherwise 
$l_{i j}=0$. The remaining decision variables are $p_{i}$, the number of points that a team $i$ has at the end of the season, and $r_{i}$, which is 1 if team $i$ is ranked higher than team $t$ at the end of the season. The highest position a team $t$ can possibly reach, given that $q$ rounds remain to be played, and that team $t$ collected $a_{t}$ points from rounds already played is given by an optimal solution of the following formulation.

minimize

$$
1+\sum_{i \in T \backslash\{t\}} r_{i}
$$

subject to

$$
\begin{aligned}
& a_{i}+q+\sum_{j \in T \backslash\{i\}: i j \in G(q)}\left(2.01 w_{i j}-l_{i j}\right) \\
& +\sum_{j \in T \backslash\{i\}: j i \in G(q)}\left(2.01 l_{j i}-w_{j i}\right)=p_{i}, \forall i \in T \\
& w_{i j}+l_{i j} \leqslant 1, \forall i j \in G(q) \\
& p_{t} \geqslant p_{i}-B r_{i}, \forall i \in T \backslash\{t\} \\
& w_{i j}, l_{i j} \in\{0,1\}, \forall i j \in G(q) \\
& r_{i} \in\{0,1\}, \forall i \in T \backslash\{t\}
\end{aligned}
$$

The goal function minimizes the number of teams that are ranked before team $t$, and is scaled with the term 1 to indicate that the highest ranking it can obtain is the first place. The first set of constraints states that the number of points a team $i$ has at the end of the season equals the points this team already has, plus 3.01 points for each win and 1 point for each draw in the games that are yet to be played. In case of an equal number of points, the team that won the highest number of games is to be ranked first. Therefore, instead of 3 , we add 3.01 points for a win. Notice that in case of a draw, both $w_{i j}$ and $l_{i j}$ equal 0 . The situation where both $w_{i j}$ and $l_{i j}$ equal 1 is not allowed by the second set of constraints. Finally, we need to make sure that a team $i$ will be ranked higher than $t$, if it obtained more points than $t$. When the parameter $B$ is chosen equal to the total number of points that can be won in the competition, the final set of constraints will do just this. This formulation can be solved with Ilog Cplex 10.2 in less than one minute.

With a limited number of changes, the above formulation can be used to determine the lowest ranking that this team $t$ could still end up with. The goal function should be changed to maximize the number of teams that has a higher ranking than team $t$. Further, constraint set (5) should be replaced by

$$
p_{t} \leqslant p_{i}+B\left(1-r_{i}\right), \forall i \in T \backslash\{t\},
$$

such that a team $i$ is only ranked higher than team $t$, if team $i$ has more points.

The above formulations allow us to determine whether a team is still competing for some outcome or not. For instance, a team is still in the running for the league title, if the highest position this team can still reach is the first, and if the lowest position this team can still drop to is lower than the first. Indeed, if the former was not the 
case, the team would no longer be able to win the championship, and if only the latter was not the case, the league title could no longer escape them. A similar reasoning can be made for the other relevant outcomes: qualification for European football and relegation. Note that Ribeiro and Urrutia (2005) developed an integer programming model to determine the minimum number of points any given team has to win in order to be sure it will reach some outcome. A similar model results in the minimum number of points this team has to win in order to have any chance of reaching that outcome. With these numbers, it is also possible to determine whether a match still has any importance or not.

Although as far as we are aware, no research has been done on the influence the schedule has on the competition, it seems reasonable that some influence exists. For sure, the schedule will have its consequences for the importance of a specific game. Indeed, a game that is scheduled in the beginning of the season can never be unimportant, whereas this same game may very well be unimportant when scheduled on the final round. It is however harder to say what the influence of the schedule on the importance of a competition, or the number of unimportant games in the competition could be. The sequence of opponents for some team may also have an influence on the importance of its games. A team that plays all the weak opponents in the beginning of the season will probably win most of these games, and will be in the race for the league title until the last rounds, despite a series of tough opponents that remain to be played. Indeed, we always compare the best case scenario, where all the tough opponents are defeated, with the worst case scenario to determine whether a game still has importance. On the other hand, a team that loses its first games, will have unimportant games (with respect to the league title) earlier in the season, because even the best case scenario will eventually no longer suffice to win the league title. However, when considering the average match importance in a competition, it is conceivable that these effects cancel out each other, since a schedule can never offer an easy start for all teams. Finally, notice that because of our choice to simulate the outcome of games independently of the outcome of previous simulated games, the influence of the schedule on the outcome of the competition (and possibly also on match unimportance) is reduced. Indeed, if a series of wins would increase the chance of winning a next game, the effect of, for instance, a good start of the season would be increased.

\section{Results}

In this section, we present the results of simulations of the four league formats discussed in section 3. For each of these formats, we simulate a series of five consecutive seasons. We think five consecutive seasons is enough to grasp the dynamics of the league composition with respect to relegation. On the other hand, the strength of the teams, as determined by historical results over 10 years may no longer be valid when simulating series of more than five consecutive seasons. As announced in section 2, for each round, we compute the importance of the matches using our measure described in section 6 , and subsequently, we use the probability distributions derived in section 5 to simulate the outcomes of the matches. When the regular competition is simulated, the play-offs to determine the teams that qualify for European football are simulated in the same way. A direct climber from the second division is selected randomly from 
a set including all clubs that are not playing in the first division at that time. A team that needs to play a play-off tournament to claim its place in the first division (as in the current league format, and the play-off league) is chosen with equal probability among the participants of this tournament. The participants of this tournament other than the one(s) coming from the first division are selected with an equal probability among the teams that did not play in the first division that season.

When possible we have used the same match results in the various league formats. This technique is known as common random numbers (we refer to Kelton et al. (2002) for more information). Since in the current league, each team will play at most one home game against each other team, we could simulate 100 series of 5 seasons, while we could only simulate 50 series for each of the other league formats, using the same match results. Indeed, in the future league and the play-off league, a team can play two home games against another team per season (and even three in the Wijnants league). Further, not all league formats have the same teams taking part (due to a different number of competitors and relegation rule), and the number of occurrences of a game can differ as well between the various league formats. Thus, we cannot point out the league format as the only factor that determines whether a team wins the league, qualifies for European football or relegates, but we have tried to limit the influence of random match results.

We made the following assumptions and simplifications. In the season 2009-2010, five clubs will qualify for European football. Two of them go to (the qualification stage of) the Champions League; the other three go to the Europa League. We assume that over the five season period used in our simulation, Belgium will maintain these five tickets for European football. Four tickets are decided through the league, but the fifth team to qualify for European football is the winner of the Belgian Cup. The Belgian Cup is a tournament that is not influenced by the way in which the league is organized, and the Cup Final is typically played after the final round of the league. Although the Cup can have an influence on the distribution of the tickets for European football in the league (e.g., if the Cup finalists end on two of the four first positions in the league, the fifth in the league qualifies for European football), and consequently also on match importance, we have not taken this into account. Further, the matches are normally played on Saturdays, but the TV station has the right to move one game to Friday, and two to Sunday. Only on the last two rounds, all games should be played simultaneously. Obviously, this can have consequences for match importance, but since there is no clear rule to determine which games will be moved to an earlier or later date, we assume that all games in the same round are played at the same time. Also, in the Wijnants league, the winner of the A series of the autumn competition qualifies for European football. This means that, from the point of view of qualification for European football, the games in the spring competition are no longer important for this team. On the other hand, if this team would end second in the spring league, its ticket for the play-off for European football goes to the third in the spring league. In order not to complicate the model, we have not taken these particularities into account, and assume that their effects on match importance will cancel out each other. Finally, since promotion/relegation tournaments fall under the authority of the second division, we have not considered these matches when comparing league formats. 
Table 3 Average match importance

\begin{tabular}{rrrrr}
\hline Outcome & Current league & Play-off league & Wijnants league & Future league \\
\hline League Champion & 0.0215 & 0.0253 & 0.0200 & 0.0261 \\
European football & 0.0174 & 0.0279 & 0.0234 & 0.0266 \\
Relegation & 0.0177 & 0.0225 & 0.0184 & 0.0163 \\
\hline
\end{tabular}

Table 3 gives an overview of the average match importance for each league format and for each outcome. The play-off league and the future league both offer a high average match importance for the league championship and qualification for European football. Moreover, the play-off league clearly outperforms the other leagues with regard to relegation. The current league has a poor average match importance; all other league formats score better on at least two of the three outcomes. Thus, with respect to match importance, any change seems to be an improvement.

Table 4 Proportion of unimportant games

\begin{tabular}{rrrrr}
\hline Outcome & Current league & Play-off league & Wijnants league & Future league \\
\hline League Champion & $17.2 \%$ & $19.1 \%$ & $31.3 \%$ & $15.6 \%$ \\
European football & $9.1 \%$ & $6.7 \%$ & $9.7 \%$ & $5.6 \%$ \\
Relegation & $7.6 \%$ & $13.3 \%$ & $29.4 \%$ & $25.5 \%$ \\
A series & - & - & $5.7 \%$ & - \\
Any outcome & $3.0 \%$ & $3.0 \%$ & $2.3 \%$ & $2.7 \%$ \\
\hline
\end{tabular}

Table 4 shows the proportion of unimportant games for each outcome and each of the league formats. The current league has a low number of unimportant games with respect to the league title and relegation. Adopting the future league will however decrease the number of games that have no importance for the league title and European football. Since relegation is settled after at most 30 of the 40 rounds, the future league has quite a lot of unimportant matches with respect to relegation. The same goes for the play-off league, where all the matches in the European play-off stage obviously do not matter for relegation (recall that the relegation/promotion play-off is considered as belonging to the second division, and thus not taken into account). Although the Wijnants league seems to give many chances to all teams to take part in the play-off for European qualification, the proportion of unimportant games for this outcome is quite high. The number of unimportant games for the league title and relegation are also quite high, which can be explained by the fact that teams in the A series of the spring competition cannot relegate to the second division, and that teams in the B series of the spring already lost their chances on the league title half-way the season. This alone results in almost $25 \%$ unimportant games for these outcomes. The Wijnants league however offers an extra objective to play for, namely promotion to (or maintaining a place in) the A series. This explains why the number of games that do not matter for any outcome is very low in this league format.

Another consideration is the number of games that a team can expect to play against the top teams per season (see Table 5). Adopting the play-off league would 
Table 5 Average number of games per season against top four teams (averaged over the members of each team group)

\begin{tabular}{rrrrr}
\hline Team group & Current league & Play-off league & Wijnants league & Future league \\
\hline Top four & 6.0 & 7.6 & 10.6 & 11.6 \\
Ambitious middle & 7.9 & 8.3 & 9.3 & 10.4 \\
Small six & 5.8 & 5.0 & 3.5 & 5.7 \\
Second division & 3.0 & 2.1 & 2.7 & 1.8 \\
\hline
\end{tabular}

Table 6 Average number of games per season, if playing in the first division (averaged over the members of each team group)

\begin{tabular}{rrrrr}
\hline Team group & Current league & Play-off league & Wijnants league & Future league \\
\hline Top four & 34.0 & 32.7 & 36.5 & 40.3 \\
Ambitious middle & 34.0 & 30.9 & 36.4 & 39.3 \\
Small six & 34.0 & 29.8 & 35.8 & 34.4 \\
Second division & 34.0 & 30.2 & 36.4 & 35.0 \\
\hline
\end{tabular}

increase the number of games against top teams for the top four teams, but only with one and a half game per season on average. On the one hand, the play-offs for European football offer possibilities for extra games against the top teams, but on the other hand, the fact that there are only 16 teams competing in this league reduces the chances to be in the league. For the ambitious middle teams the result is a modest increase, but the small six and the second division teams will play less games against top teams than in the current league. The Wijnants league and the future league offer a spectacular increase in the number of games per season against top teams, for the top four and the ambitious middle teams. With the Wijnants league, this goes at the expense of the smaller clubs, whereas in the future league, only the second division teams are worse off. The downside of this increased number of games against top teams is that the season is considerably longer in the future league (see Table 6). Indeed, a typical season in first division future league will consist of around 40 matches for top teams and ambitious middle teams, although only 16 teams compete in this league. The only league format that manages to reduce the number of matches compared to the current league is the play-off league. Notice that since we did not take into account the relegation/promotion tournament, the number of games actually played per season will be higher than indicated in Table 6 for teams involved in relegation.

Table 7 Percentage of presence in simulated first division seasons (averaged for each team group)

\begin{tabular}{rrrrr}
\hline Team group & Current league & Play-off league & Wijnants league (A+B) & Future league (1-6,7-12) \\
\hline Top four & $100.0 \%$ & $100.0 \%$ & $100.0 \%(93.9 \%+6.1 \%)$ & $100.0 \%(96.6 \%, 3.3 \%)$ \\
Ambitious middle & $98.5 \%$ & $94.3 \%$ & $97.1 \%(57.7 \%+39.4 \%)$ & $96.7 \%(31.8 \%, 55.0 \%)$ \\
Small six & $72.4 \%$ & $62.0 \%$ & $76.1 \%(20.7 \%+55.4 \%)$ & $67.8 \%(2.8 \%, 28.8 \%)$ \\
Second division & $37.5 \%$ & $26.2 \%$ & $56.1 \%(15.4 \%+40.7 \%)$ & $21.3 \%(0.6 \%, 8.4 \%)$ \\
\hline
\end{tabular}


The main concern of the small six and the second division teams is that the new league formats will reduce their chances of playing in the first division. Therefore, we computed for each team the percentage of the simulated first division seasons in which they participated, and averaged these results for each team group. The results can be found in Table 7 and show that the small six and the second division teams indeed play less frequently in the first division with the play-off league and the future league, which has two teams less than the current league. However, we notice that the ambitious middle group may also expect to suffer somewhat from the reduced number of participants in this league. The Wijnants league has 20 teams, which explains the increased presence for all teams compared with the current league. However, the small six and the second division teams will mostly play in the (less interesting) B series, although the average top team will also play in the B series for $6 \%$ of the time. As was to be expected, the second stage of the future league tournament with teams ranked 1-6 will be dominated by top clubs and is less accessible for the other teams than the A series of the Wijnants league.

Table 8 Distribution of the league champion over the team groups

\begin{tabular}{rrrrr}
\hline Team group & Current league & Play-off league & Wijnants league & Future league \\
\hline Top four & $99.8 \%$ & $98.8 \%$ & $96.4 \%$ & $98.8 \%$ \\
Ambitious middle & $0.2 \%$ & $1.2 \%$ & $3.6 \%$ & $1.2 \%$ \\
Small six & $0.0 \%$ & $0.0 \%$ & $0.0 \%$ & $0.0 \%$ \\
Second division & $0.0 \%$ & $0.0 \%$ & $0.0 \%$ & $0.0 \%$ \\
\hline
\end{tabular}

Table 8 shows how the league champion is distributed over the team groups. The influence of the league formats on the league title is minimal. Only the Wijnants league offers a slightly larger chance to ambitious middle teams. The balance of power among the Belgian football teams seems to be such that the top teams rarely lose a championship, regardless of the league format.

Table 9 Distribution of qualification for European football over the team groups

\begin{tabular}{rrrrr}
\hline Team group & Current league & Play-off league & Wijnants league & Future league \\
\hline Top four & $87.3 \%$ & $78.7 \%$ & $78.4 \%$ & $79.4 \%$ \\
Ambitious middle & $12.5 \%$ & $20.4 \%$ & $18.5 \%$ & $18.8 \%$ \\
Small six & $0.2 \%$ & $0.8 \%$ & $1.3 \%$ & $1.4 \%$ \\
Second division & $0.1 \%$ & $0.1 \%$ & $1.8 \%$ & $0.4 \%$ \\
\hline
\end{tabular}

The consequences of a change in league format for qualification for European football are however considerable. Table 9 shows the chances for qualification for European football; tables 10 and 11 show how the tickets for respectively the Champions League and the Europa League are distributed. Any change in format seriously reduces the chances of qualifying for European football for the top teams. The difference is largely pulled in by the ambitious middle teams. The Champions League remains a privilege 
Table 10 Distribution of qualification for Champions league football over the team groups

\begin{tabular}{rrrrr}
\hline Team group & Current league & Play-off league & Wijnants league & Future league \\
\hline Top four & $97.6 \%$ & $87.6 \%$ & $93.2 \%$ & $96.0 \%$ \\
Ambitious middle & $2.4 \%$ & $11.8 \%$ & $6.6 \%$ & $4.0 \%$ \\
Small six & $0.0 \%$ & $0.6 \%$ & $0.0 \%$ & $0.0 \%$ \\
Second division & $0.0 \%$ & $0.0 \%$ & $0.2 \%$ & $0.0 \%$ \\
\hline
\end{tabular}

Table 11 Distribution of qualification for Europa League football over the team groups

\begin{tabular}{rrrrr}
\hline Team group & Current league & Play-off league & Wijnants league & Future league \\
\hline Top four & $77.0 \%$ & $69.8 \%$ & $63.6 \%$ & $62.8 \%$ \\
Ambitious middle & $22.4 \%$ & $29.0 \%$ & $30.4 \%$ & $33.6 \%$ \\
Small six & $0.3 \%$ & $1.0 \%$ & $2.6 \%$ & $2.8 \%$ \\
Second division & $0.2 \%$ & $0.2 \%$ & $3.4 \%$ & $0.8 \%$ \\
\hline
\end{tabular}

for top teams, although the play-off league also offers a fair chance to ambitious middle teams. Compared to the current league, the top teams also lose a share of Europa League tickets in any other league format, although this time, the small six and even the second division teams also benefit from this.

In general, the results show that there is no league format that outperforms the other formats on all aspects. The current league however does not perform very well. All other leagues are expected to be more attractive, since typically less games will be unimportant, and the average match importance will usually be higher. An increased number of games against top teams for the top teams and the ambitious middle is to be expected especially from the future league and the Wijnants league. These are the main concern for the TV station. Furthermore, the current league seems to quite beneficial for the top teams when we consider the league title and qualification for European football. All new leagues formats will increase the chances for European football for the ambitious middle group, at the expense of the top teams. The playoff league and the future league however decreases the chances of playing in the first division for the small six and the second division teams. The opposite is true for the Wijnants league, although in this league those teams would mostly play in the B series.

\section{Conclusions and future work}

In this paper, we compared the current league format with the future league format and two other formats that have been under consideration. We have presented tools to evaluate the attractiveness of these league formats by measuring the match importance that is to be expected. Further, we developed a model based on historical match results to simulate the outcome of each of these formats. This allowed us to estimate the number of seasons a team would play in the first division, its number of games against top teams, and its chances for winning the league or qualifying for European football. 
During this research, the debate on the reform of the league in Belgian football was ongoing. Although we were not directly involved in this discussion, we had the impression that - apart from the different interests of all involved parties - one of the main problems was the lack of a study on what can be expected from a new league format. Eventually, the Royal Belgian Football Association decided to select the format that we refer to as the future league from the season 2009-2010 onwards. The results show that this decision will indeed increase the average match importance and reduce the number of unimportant matches for all outcomes except relegation, which is exactly what the TV station wanted. The level of the competition should increase, since the top teams and the ambitious middle group will play significantly more top games. On the other hand, the season will also be longer, which could be wearying for those teams competing in European competitions as well. The fact that two teams less will play in the first division will halve the chances of the second division teams to play in the first division, but will have few consequences on the chances of playing in the first division for the other teams. We expect that the top teams will still win almost every league title, but they will lose ground with respect to qualification for European football. More specifically, teams from the ambitious middle will qualify more often for the Europa League, at the expense of a top team.

This topic leaves space for some future research. It would be very interesting to compare our measure for match importance with other measures, as e.g., the one by Schilling (1994). Further, research could be done to investigate the influence of the schedule on match importance. Finally, linking this research to the expected number of spectators would make the financial aspects of adopting a new league format more tangible.

\section{References}

Audas R, Dobson S, Goddard J (2002) The impact of managerial change on team performance in professional sports. Journal of Economics and Business 54(6):633650

Bartsch T, Drexl A, Kroger S (2006) Scheduling the professional soccer leagues of Austria and Germany. Computers and Operations Research 33(7):1907-1937

Bojke C (2007) The impact of post-season play-off systems on the attendance at regular season games. In: Albert, J., Koning, R.H. (eds.) Statistical Thinking in Sports, pp 179-202

Colin F (2007) AA Gent en Charleroi leiden verzet tegen competitiehervorming (in Dutch). De Standaard, October 24th

de Werra D (1980) Geography, games and graphs. Discrete Applied Mathematics 2(4):327-337

Della Croce F, Oliveri D (2006) Scheduling the Italian Football League: an ILP-based approach. Computers and Operations Research 33(7):1963-1974

De Vos G (2008a) De kronkels van de competitiehervorming (in Dutch). De Standaard, March 19th

De Vos G (2008b) Misbaksel (in Dutch). De Standaard, March 22nd

Dixon M, Coles S (1997) Modelling association football scores and inefficiencies in the football betting market. Applied Statistics 46:265-280 
Dixon M, Pope P (2004) The value of statistical forecasts in the UK association football betting market. International Journal of Forecasting 20:686-700

Durán G, Guajardo M, Miranda J, Sauré D, Souyris S, Weintraub A (2007) Scheduling the Chilean Soccer League by integer programming. Interfaces 37:539-552

Forrest D, Simmons R (2000) Forecasting sport: the behaviour and performance of football tipsters. International Journal of Forecasting 16:317-331

Geril J (2008a) Competitie met 16 goedgekeurd (in Dutch). De Standaard, May 19th

Geril J (2008b) Naar een competitie met 16 (in Dutch). De Standaard, January 18th

Goddard J (2005) Regression models for forecasting goals and match results in association football. International Journal of Forecasting 21:331-340

Goddard J, Asimakopoulos I (2004) Forecasting football match results and the efficiency of fixed-odds betting. Journal of Forecasting 23:51-66

Goossens D, Spieksma F (2009) Scheduling the Belgian soccer league. Interfaces 39(2):109-118.

Goossens D, Beliën J, Spieksma F (2008) Comparing league formats with respect to match unimportance in Belgian football. In: Proceedings of the 7th International Conference on the Practice and Theory of Automated Timetabling (PATAT 2008), Montréal (Canada), August 18-22.

Griggs TS, Rosa A (1996) A tour of European soccer schedules, or testing the popularity of $G K_{2 n}$. Bulletin of the Institute of Combinatorics and its Applications 18:65-68

Hauspie J (2007) Het plan-Wijnants voor de hervorming van de Jupiler League: simpele rekensom (in Dutch). Sport Voetbalmagazine 7(48):24-25

Jennett N (1984) Attendances, uncertainty of outcome and policy in Scottish league football. Scottish Journal of Political Economy 31:176-198

Karlis D, Ntzoufras I (2003) Analysis of sports data by using bivariate Poisson models. Statistician 52:381-393

Kelton WD, Sadowski RP, Sadowski DA (2002) Simulation with Arena. McGraw-Hill, Inc., New York, NY, USA

Kendall G (2008) English football fixtures over holiday periods. Journal of the Operational Research Society 59:743-755

Kendall G, Knust S, Ribeiro CC, Urrutia S (2010) Scheduling in Sports: An Annotated Bibliography. Computers and Operations Research 37:1-19

Koning R (2000) Balance in competition in Dutch soccer. Statistician 49:419-431

Koning R (2007) Post-season play and league design in Dutch soccer. In: Rodriguez, P., Garcia, J., Kesenne, S. (eds.) League Governance, Competition and Professional Sports, pp 183-207

Koning R, Koolhaas M, Renes G, Ridder G (2003) A simulation model for football championships. European Journal of Operational Research 148(2):268-276

McHale I, Scarf P (2006) Forecasting international soccer match results using bivariate discrete distributions. Working paper, Salford Business School

Rasmussen R (2008) Scheduling a triple round robin tournament for the best Danish soccer league. European Journal of Operational Research 185(2):795-810

Ribeiro C, Urrutia S (2005) An application of integer programming to playoff elimination in football championships. International Transactions in Operational Research 12:375-386

Scarf PA, Shi X (2008) The importance of a match in a tournament. Computers and Operations Research 35(7):2406-2418

Schilling MF (1994) The importance of a game. Mathematics Magazine 67(4):282-288 
Silva C, Garcia E, Saliby E (2002) Soccer championship analysis using Monte Carlo simulation. In: Yücesan, E., Chen, C.H., Snowdon, J.L. and Charnes, J.M. (Eds.). Proceedings of the Winter Simulation Conference, 8-11 Dec. 2002 (to appear), vol 2, pp 2011-2016

Smyth DJ, Smyth SJ (1994) Major league baseball division standings, sports journalists' predictions and player salaries. Managerial and Decision Economics 15(5):421429 